\title{
Subsolidus Phase Relationships in Part of the System Si, Al, Ca/N, O
}

\author{
W. Y. Sun, T. S. Yen (D. S. Yan) \\ Shanghai Institute of Ceramics, Academia Sinica, Shanghai, \\ People's Republic of China \\ \&
}

T. Y. Tien

The University of Michigan, Ann Arbor, Michigan 48109, USA

(Received 7 September 1987; accepted 22 September 1987)

\begin{abstract}
Subsolidus phase relationships in the region bounded by $\mathrm{Si}_{3} \mathrm{~N}_{4}$, $\mathrm{SiO}_{2}, \mathrm{CaSiO}_{3}, 2 \mathrm{CaO} . \mathrm{Al}_{2} \mathrm{O}_{3} . \mathrm{SiO}_{2}, \mathrm{CaO} . \mathrm{Al}_{2} \mathrm{O}_{3}, \mathrm{Al}_{2} \mathrm{O}_{3}$ and $\beta^{\prime}-\mathrm{Si}_{2} \mathrm{Al}_{4} \mathrm{O}_{4} \mathrm{~N}_{4}\left(\beta_{60}\right)$ have been studied. A new quinary phase with composition near to $\mathrm{CaO}$. $1 \cdot 33 \mathrm{Al}_{2} \mathrm{O}_{3} \cdot 0 \cdot 67 \mathrm{Si}_{2} \mathrm{~N}_{2} \mathrm{O}$ (designated as $\mathrm{S}$-phase) and a complete series of solid solution between $\mathrm{S}$-phase and $\mathrm{CaO} .2 \mathrm{Al}_{2} \mathrm{O}_{3}$ were found. Fourteen compatible tetrahedra, of which five contain S-phase, occur in the region explored. They are as follows: $\mathrm{X}_{1}-\mathrm{SiO}_{2}$-anorthite-mullite; $\mathrm{X}_{1}$-anorthite-mullite- $\mathrm{Al}_{2} \mathrm{O}_{3}$; $\mathrm{X}_{1}$-anorthite- $\mathrm{Al}_{2} \mathrm{O}_{3}-\beta_{60} ; \mathrm{X}_{1}$-anorthite- $\beta_{60}-\mathrm{Si}_{3} \mathrm{~N}_{4} ; \mathrm{X}_{1}$-anorthite- $\mathrm{Si}_{3} \mathrm{~N}_{4}-\mathrm{Si}_{2} \mathrm{~N}_{2} \mathrm{O}$; $\mathrm{X}_{1}$-anorthite- $\mathrm{Si}_{2} \mathrm{~N}_{2} \mathrm{O}-\mathrm{SiO}_{2} ;$ anorthite- $\mathrm{Si}_{2} \mathrm{~N}_{2} \mathrm{O}-\mathrm{SiO}_{2}-\mathrm{CaSiO}_{3}$; anorthite- $\mathrm{Si}_{2} \mathrm{~N}_{2} \mathrm{O}$ $\mathrm{CaSiO}_{3}$-gehlenite; anorthite- $\mathrm{Si}_{2} \mathrm{~N}_{2} \mathrm{O}$-gehlenite- $\mathrm{Si}_{3} \mathrm{~N}_{4}$; S-anorthite- $\mathrm{Al}_{2} \mathrm{O}_{3}-\beta_{60}$; $\mathrm{S}-\mathrm{Al}_{2} \mathrm{O}_{3}-\mathrm{CaO} .2 \mathrm{Al}_{2} \mathrm{O}_{3}$-gehlenite; $\mathrm{S}-\mathrm{Al}_{2} \mathrm{O}_{3}$-gehlenite-anorthite; S-gehlenite-anorthite- $\mathrm{Si}_{3} \mathrm{~N}_{4} ; \mathrm{S}$-anorthite- $\mathrm{Si}_{3} \mathrm{~N}_{4}-\beta_{60}$.
\end{abstract}

\section{INTRODUCTION}

Various metal oxides (e.g. $\mathrm{Al}_{2} \mathrm{O}_{3}, \mathrm{Y}_{2} \mathrm{O}_{3}$ and $\mathrm{MgO}$ ) have been proved to be effective additives to promote densification of $\mathrm{Si}_{3} \mathrm{~N}_{4}$ ceramics. Among these, only $\mathrm{Al}$ can enter into the lattice of $\beta-\mathrm{Si}_{3} \mathrm{~N}_{4}$ to form $\beta^{\prime}$-sialon with oxygen replacing nitrogen simultaneously, while the other oxides always react with the $\mathrm{SiO}_{2}$ existing at the surface of $\mathrm{Si}_{3} \mathrm{~N}_{4}$ grains and other impurities to form a glassy phase at the grain boundaries, which impairs the hightemperature mechanical properties of $\mathrm{Si}_{3} \mathrm{~N}_{4}$ ceramics. In order to crystallize out this glassy phase as second crystalline phases at the grain boundaries, it is necessary to know the subsolidus relationships in the $\mathrm{Si}, \mathrm{Al}, \mathrm{M} / \mathrm{N}, \mathrm{O}$ systems (where $\mathrm{M}$ represents metal atoms). For instance, the subsolidus relationships in the system $\mathrm{Si}, \mathrm{Al}, \mathrm{Y} / \mathrm{N}, \mathrm{O}$ have shown that garnet $\left(3 \mathrm{Y}_{2} \mathrm{O}_{3} \cdot 5 \mathrm{Al}_{2} \mathrm{O}_{3}\right)$ can be formed as a crystalline second phase of the grain boundaries. ${ }^{1-3}$ In fact, $\beta^{\prime}$-YAG ceramics with high strength at temperatures in excess of $1000^{\circ} \mathrm{C}$ have been developed. Calcium is one of the common impurities present in $\mathrm{Si}_{3} \mathrm{~N}_{4}$ powder, which persists at the grain boundaries in $\mathrm{Si}_{3} \mathrm{~N}_{4}$ ceramiç in the form of glassy phase. So, it is felt necessary to study the subsolidus phase equilibrium in the system $\mathrm{Si}, \mathrm{Al}, \mathrm{Ca} / \mathrm{N}, \mathrm{O}$ in order to develop $\beta-\mathrm{Si}_{3} \mathrm{~N}_{4}$-based ceramics from a lower quality $\mathrm{Si}_{3} \mathrm{~N}_{4}$ powder containing $\mathrm{Ca}$ as the main impurity. The compositions investigated were restricted to the region bounded by $\mathrm{Si}_{3} \mathrm{~N}_{4}, \mathrm{SiO}_{2}$, $\mathrm{CaSiO}_{3}, 2 \mathrm{CaO} . \mathrm{Al}_{2} \mathrm{O}_{3} . \mathrm{SiO}_{2}, \mathrm{CaO} . \mathrm{Al}_{2} \mathrm{O}_{3}, \mathrm{Al}_{2} \mathrm{O}_{3}$ 


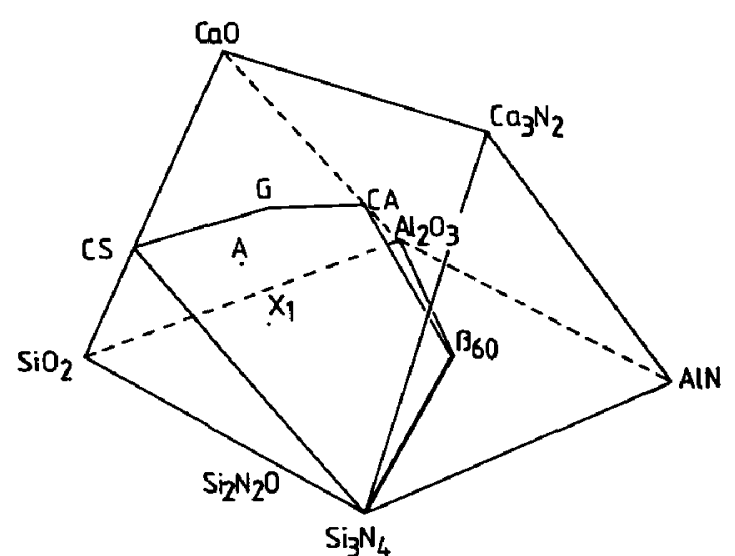

Fig. 1. The Jänecke prism for Ca-sialon system showing the region studied.

and $\beta_{60}$, as shown in Fig. 1, which was considered to involve $\mathrm{Ca}$-containing compounds that may coexist with $\mathrm{Si}_{3} \mathrm{~N}_{4}$ and $\beta^{\prime}$-sialon and is more relevant to what we would like to focus upon.

\section{EXPERIMENTAL}

The starting powders used were silicon nitride (AME, UK, containing $1.3 \%$ oxygen), aluminum nitride (Tokyo Shibaura Electric Co., Japan, containing $1.4 \%$ oxygen), calcium oxide (calcined calcium carbonate, $>99.9 \%$ ), aluminum oxide (decomposed ammonia alum, $>99.9 \%$ ) and silicon oxide (gelatinoid silica, $>99.99 \%$ ). The oxygen content of the nitride powders was taken into account in computing the various compositions. These compositions were mixed in agate mortar under absolute alcohol for $2 \mathrm{~h}$, dried and then isostatically pressed (400 MPa). Some compositions were prepared from synthesized compounds, such as $\mathrm{CaO} \cdot \mathrm{Al}_{2} \mathrm{O}_{3} \cdot 2 \mathrm{SiO}_{2}, \quad 2 \mathrm{CaO} \cdot \mathrm{Al}_{2} \mathrm{O}_{3} \cdot \mathrm{SiO}_{2}$, $\mathrm{CaO} . \mathrm{Al}_{2} \mathrm{O}_{3}$ or $\mathrm{CaO} .6 \mathrm{Al}_{2} \mathrm{O}_{3}$ etc., in order to check the results obtained from the starting powders mentioned above. Each composition was fired or hot-pressed at two different temperatures and then annealed by subsequent heat treatment at $1300^{\circ} \mathrm{C}$ for $20 \mathrm{~h}$.

All as-fired and annealed specimens were analyzed by X-ray diffraction.

\section{RESULTS AND DISCUSSION}

Twenty nine compositions were studied with different conditions of heat treatment. Their weight losses and crystalline phases present were determined and some of these are given in Table 1. Equilibrium was assumed to have been attained when the number and type of phases did not change with different heat treatments or from different starting materials. The experimental results obtained indicate that in the specimens containing large amounts of silicon nitride, some of the $\alpha-\mathrm{Si}_{3} \mathrm{~N}_{4}$ phase could not be totally transformed to $\beta^{\prime}-\mathrm{Si}_{3} \mathrm{~N}_{4}$ even at $1700^{\circ} \mathrm{C}$. Otherwise, equilibrium was readily attained in most of the compositions at $1550^{\circ} \mathrm{C}$ within one hour of firing time. Some compositions bloated at temperatures around $1550^{\circ} \mathrm{C}$ and could reach equilibrium only at temperatures below $1550^{\circ} \mathrm{C}$. Because of the weight losses observed, the phase assemblages as determined by X-ray analyses were taken as an indication of the final specimens' compositions and were used for the determination of phase relationships.

The quasi-quaternary system $\mathrm{Si}, \mathrm{Al}, \mathrm{Ca} / \mathrm{N}, \mathrm{O}$ has three independent composition variables. Therefore, the subsolidus phase relations in the system can be represented in a triangular prism (Fig. 1), in which a four-phased field can be represented as a tetrahedron. The compatibility relationships found in this system are listed in Table 2 . Graphic representation of these tetrahedra are given in Figs 2-9.

A new quinary phase (designated as S-phase) and a complete series of solid solutions between the new phase and $\mathrm{CaO} \cdot 2 \mathrm{Al}_{2} \mathrm{O}_{3}$ were found. The X-ray diffraction patterns of them are given in Table 3 and compared with the pattern of $\mathrm{CaO} \cdot 2 \mathrm{Al}_{2} \mathrm{O}_{3}$. Because of the $\mathrm{X}$-ray pattern similarity and the formation of a continuous solid solution with $\mathrm{CaO} \cdot 2 \mathrm{Al}_{2} \mathrm{O}_{3}$, $\mathrm{S}$-phase was considered to be a substituted $\mathrm{CaO} .2 \mathrm{Al}_{2} \mathrm{O}_{3}$ with $\mathrm{Si}-\mathrm{N}$ replacing $\mathrm{Al}-\mathrm{O}$ pairs. Therefore several compositions around the composition of $\mathrm{CaO} . \mathrm{Al}_{2} \mathrm{O}_{3} \cdot \mathrm{Si}_{2} \mathrm{~N}_{2} \mathrm{O}$ have been examined. The results are shown in Table 4 . It can be seen that single phase material can be obtained up to the composition $\mathrm{CaO} \cdot 1 \cdot 33 \mathrm{Al}_{2} \mathrm{O}_{3} \cdot 0 \cdot 67 \mathrm{Si}_{2} \mathrm{~N}_{2} \mathrm{O}$ (No. 4). All melted or partly melted compositions including No. 4 consisted of glass, $\alpha-\mathrm{Si}_{3} \mathrm{~N}_{4}$ and polytypoid phases such as $12 \mathrm{H}$ and $15 \mathrm{R}$. After devitrification, all compositions, even No. 4, crystallized out as S-phase plus gehlenite. This strange behaviour of No. 4 which is shown to be a pure S-phase after being directly fired to $1400^{\circ} \mathrm{C}$, may be caused by the fact that the liquid phase once formed cannot be completely devitrified. So it seems that solid state reaction is necessary to get single-phase S-phase. Compositions 1,4 and 5 are located on the line between $\mathrm{CaO}: 2 \mathrm{Si}_{2} \mathrm{~N}_{2} \mathrm{O}$ and $\mathrm{CaO}: 2 \mathrm{Al}_{2} \mathrm{O}_{3}$. From Xray analyses, it is obvious that the limit of solubility is about $\mathrm{CaO} \cdot 1 \cdot 33 \mathrm{Al}_{2} \mathrm{O}_{3} \cdot 0 \cdot 67 \mathrm{Si}_{2} \mathrm{~N}_{2} \mathrm{O}$.

$\mathrm{CaO} .6 \mathrm{Al}_{2} \mathrm{O}_{3}$ was very difficult to form in this 
Table 1. Experimental data used to establish phase relationships.

\begin{tabular}{|c|c|c|c|c|c|c|c|c|c|c|}
\hline \multirow[t]{2}{*}{ No. } & \multicolumn{5}{|c|}{ Composition (eq. \%) } & \multirow{2}{*}{$\begin{array}{l}\text { Temp. } \\
\left({ }^{\circ} \mathrm{C}\right)\end{array}$} & \multirow{2}{*}{$\begin{array}{l}\text { Time } \\
\text { (h) }\end{array}$} & \multirow{2}{*}{$\begin{array}{l}\text { W.L. } \\
(\%)\end{array}$} & \multirow{2}{*}{ Phase present } & \multirow[t]{2}{*}{ Note } \\
\hline & $\mathrm{Si}$ & $A \mid$ & $\mathrm{Ca}$ & 0 & $\mathbf{N}$ & & & & & \\
\hline 2 & $54 \cdot 29$ & 42.59 & $3 \cdot 12$ & $59 \cdot 64$ & $40 \cdot 36$ & $\begin{array}{l}1550 \\
1300 \\
1700 \\
1300\end{array}$ & $\begin{array}{r}1 \\
20 \\
1 \\
20\end{array}$ & $\begin{array}{r}3 \cdot 6 \\
17 \cdot 5\end{array}$ & $\begin{array}{l}\text { AlOs, B30w, Bw, aw } \\
\text { AlOs, Am, Bmw, B30w, aw, X1www } \\
\text { B50vs, AlOmw, X1w } \\
\text { B50vs, Am, X1w, AlOvw }\end{array}$ & \\
\hline 3 & $83 \cdot 68$ & $12 \cdot 55$ & 3.77 & $30 \cdot 96$ & 69.04 & $\begin{array}{l}1550 \\
1300 \\
1700 \\
1300\end{array}$ & $\begin{array}{r}1 \\
20 \\
1 \\
20\end{array}$ & $\begin{array}{l}2 \cdot 5 \\
1 \cdot 7\end{array}$ & $\begin{array}{l}\text { Bs, } \alpha \mathrm{ms}, \text { AlOmw } \\
\text { Bvs, } \alpha s, \text { Am } \\
\text { Bvs, AlOm } \\
\text { Bvs, Aw, AlOvw }\end{array}$ & $\begin{array}{l}A^{b} \\
A^{b}\end{array}$ \\
\hline 4 & $79 \cdot 43$ & $9 \cdot 41$ & $11 \cdot 16$ & $81 \cdot 19$ & $18 \cdot 81$ & $\begin{array}{l}1550 \\
1300 \\
1700 \\
1300\end{array}$ & $\begin{array}{r}1 \\
20 \\
1 \\
20\end{array}$ & $\begin{array}{l}12 \cdot 3 \\
18 \cdot 7\end{array}$ & $\begin{array}{l}\text { SNOm, Bvw } \\
\text { SNOm, Bvvw } \\
\text { SNOm, Bvw, avw } \\
\text { SNOm, Bvw, avw }\end{array}$ & \\
\hline 5 & $61 \cdot 64$ & $20 \cdot 20$ & $18 \cdot 16$ & $81 \cdot 15$ & $18 \cdot 85$ & $\begin{array}{l}1550 \\
1300 \\
1700 \\
1300\end{array}$ & $\begin{array}{r}1 \\
20 \\
1 \\
20\end{array}$ & $\begin{array}{c}11 \cdot 6 \\
/\end{array}$ & $\begin{array}{l}\text { Bvw, avvw } \\
\text { Gvs, As, SNOmw, Bvw, avw, Cvw } \\
\text { Bvvw. } \\
\text { Gs, SNOm, Amw, Cwvw }\end{array}$ & \\
\hline 6 & $73 \cdot 81$ & $10 \cdot 71$ & $15 \cdot 48$ & $56 \cdot 25$ & $43 \cdot 75$ & $\begin{array}{l}1550 \\
1300 \\
1450 \\
1300\end{array}$ & $\begin{array}{r}1 \\
20 \\
1 \\
20\end{array}$ & $\begin{array}{l}11 \cdot 4 \\
10 \cdot 1\end{array}$ & 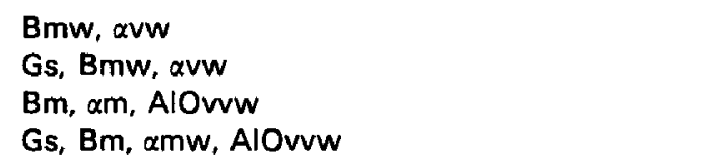 & \\
\hline 8 & $36 \cdot 79$ & 60.09 & $3 \cdot 12$ & $79 \cdot 64$ & $20 \cdot 36$ & $\begin{array}{l}1550 \\
1300 \\
1700 \\
1300\end{array}$ & $\begin{array}{r}1 \\
20 \\
1 \\
20\end{array}$ & $\begin{array}{l}2 \cdot 2 \\
/\end{array}$ & $\begin{array}{l}\text { AlOvs, Bw, } \alpha w, B 30 v w \\
\text { AlOs, Am, X1w, Bw, } \alpha w, B 30 v w \\
\text { AlOs, B60m } \\
\text { AlOs, B60mw, Amw, X1vvw }\end{array}$ & \\
\hline 11 & $62 \cdot 15$ & $27 \cdot 58$ & $10 \cdot 27$ & $55 \cdot 00$ & $45 \cdot 00$ & $\begin{array}{l}1550 \\
1300 \\
1500 \\
1300\end{array}$ & $\begin{array}{r}1 \\
20 \\
1 \\
20\end{array}$ & $\begin{array}{l}1 \\
5 \cdot 3\end{array}$ & 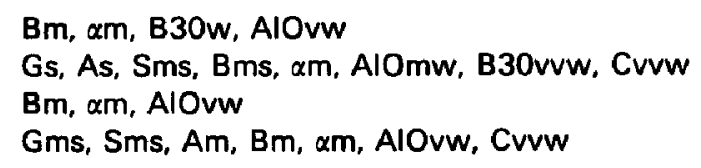 & \\
\hline 12 & $67 \cdot 20$ & $24 \cdot 24$ & $8 \cdot 56$ & $46 \cdot 67$ & $53 \cdot 33$ & $\begin{array}{l}1550 \\
1300 \\
1450 \\
1300\end{array}$ & $\begin{array}{r}1 \\
20 \\
1 \\
20\end{array}$ & $\begin{array}{l}6 \cdot 4 \\
6 \cdot 3\end{array}$ & $\begin{array}{l}\text { B30m, Bm, } \alpha m, A 10 v w \\
\text { Ams, Sm, Gm, Bm, } \alpha m, \text { B30mw, AlOw } \\
\text { Bm, B30mw, } \alpha m w, A 1 O m w \\
\text { Am, Bmw, Smw, Gw, } \alpha w, \text { AlOww }\end{array}$ & \\
\hline 13 & $50 \cdot 13$ & $37 \cdot 60$ & $12 \cdot 26$ & $62 \cdot 40$ & $37 \cdot 60$ & $\begin{aligned} 1550 \\
1300 \\
\text { HP } 1450 \\
1300\end{aligned}$ & $\begin{array}{c}1 \\
20 \\
0.5 \\
20\end{array}$ & $\begin{array}{l}4 \cdot 2 \\
/\end{array}$ & $\begin{array}{l}\text { Sw, Bw, avvw, AlOvvw } \\
\text { Ss, Gm, Aw, Bw, AlOvw } \\
\text { Sw, Bw, } \alpha w, A I O v w \\
\text { Ss, Gms, Amw, Bmw, avw, AlOwww }\end{array}$ & \\
\hline
\end{tabular}


Table 1-contd.

\begin{tabular}{|c|c|c|c|c|c|c|c|c|c|c|}
\hline \multirow[t]{2}{*}{ No. } & \multicolumn{5}{|c|}{ Composition (eq. \%) } & \multirow{2}{*}{$\begin{array}{l}\text { Temp. } \\
\left({ }^{\circ} \mathrm{C}\right)\end{array}$} & \multirow{2}{*}{$\begin{array}{l}\text { Time } \\
\text { (h) }\end{array}$} & \multirow{2}{*}{$\begin{array}{l}\text { W.L. } \\
(\%)\end{array}$} & \multirow[t]{2}{*}{ Phase present } & \multirow[t]{2}{*}{ Note } \\
\hline & Si & Al & $\mathrm{Ca}$ & 0 & $\mathbf{N}$ & & & & & \\
\hline 14 & $32 \cdot 24$ & $53 \cdot 31$ & $14 \cdot 44$ & $90 \cdot 60$ & $9 \cdot 40$ & $\begin{array}{l}1550 \\
1300 \\
1550 \\
1300\end{array}$ & $\begin{array}{r}1 \\
20 \\
1 \\
20\end{array}$ & $\begin{array}{c}7 \cdot 3 \\
6\end{array}$ & $\begin{array}{l}\text { AlOm } \\
\text { Gs, AlOm, Am, Sw, Crvw } \\
\text { AlOs } \\
\text { GS, A1Oms, Am, Sm, Cvvw }\end{array}$ & $A^{b} \cdot C 6 A^{b}$ \\
\hline 15 & $42 \cdot 99$ & $42 \cdot 99$ & $14 \cdot 03$ & $89 \cdot 25$ & $10 \cdot 75$ & $\begin{array}{l}1550 \\
1300 \\
1450 \\
1300\end{array}$ & $\begin{array}{r}1 \\
20 \\
1 \\
20\end{array}$ & $\begin{array}{r}9 \cdot 1 \\
10 \cdot 1\end{array}$ & $\begin{array}{l}\text { AlOvvw } \\
\text { As, Ss, Gm, AlOvw, Cvvw } \\
\text { AlOs } \\
\text { Ams, Gm, Sw, AlOw, Crvw }\end{array}$ & \\
\hline 16 & 34.05 & $54 \cdot 66$ & $11 \cdot 29$ & $94 \cdot 63$ & $5 \cdot 73$ & $\begin{array}{l}1550 \\
1300 \\
1500 \\
1300\end{array}$ & $\begin{array}{r}1 \\
20 \\
1 \\
20\end{array}$ & $\begin{array}{l}8 \cdot 4 \\
8 \cdot 4\end{array}$ & $\begin{array}{l}\text { AlOs } \\
\text { AlOms, Am, Gm, Svw, Crvw } \\
\text { AlOvs, Avw } \\
\text { AlOms, Gm, Am, Sw, Crw }\end{array}$ & \\
\hline 18 & $36 \cdot 96$ & $52 \cdot 97$ & $10 \cdot 07$ & $92 \cdot 54$ & $7 \cdot 46$ & $\begin{array}{l}1550 \\
1300 \\
1700 \\
1300\end{array}$ & $\begin{array}{r}1 \\
20 \\
1 \\
20\end{array}$ & $\begin{array}{l}5 \cdot 9 \\
11\end{array}$ & $\begin{array}{l}\text { AlOs } \\
\text { AlOms, Ams, Gw, Sw, Cvw, Bvvw } \\
\text { AlOw } \\
\text { AlOm, Am, Gw, Sw, Cvvw, Bvvw }\end{array}$ & \\
\hline 19 & $25 \cdot 07$ & $61 \cdot 78$ & $13 \cdot 15$ & $81 \cdot 20$ & $18 \cdot 80$ & $\begin{aligned} 1550 \\
1300 \\
\text { HP } 1450 \\
1300\end{aligned}$ & $\begin{array}{c}1 \\
20 \\
0 \cdot 5 \\
20\end{array}$ & $\begin{array}{l}4 \cdot 4 \\
/\end{array}$ & $\begin{array}{l}\text { AlOm, Sw } \\
\text { Gms, AlOm, S'mw, Avw } \\
\text { S's, AlOw, Gvw } \\
\text { S's, AlOw, Gvw }\end{array}$ & $\mathrm{C} 2 \mathrm{~A}^{b}$ \\
\hline 20 & $39 \cdot 64$ & $40 \cdot 35$ & $20 \cdot 19$ & $81 \cdot 17$ & $18 \cdot 83$ & $\begin{array}{l}1550 \\
1300 \\
1450 \\
1300\end{array}$ & $\begin{array}{r}1 \\
20 \\
1 \\
20\end{array}$ & $\begin{array}{r}5 \cdot 8 \\
11 \cdot 1\end{array}$ & $\begin{array}{l}\text { None } \\
\text { Gs, Sm, Aw, AlOvw } \\
\text { Gs } \\
\text { Gs, Smw, Aw, AlOvw }\end{array}$ & \\
\hline 29 & $74 \cdot 21$ & $19 \cdot 67$ & $6 \cdot 10$ & $25 \cdot 35$ & $74 \cdot 65$ & $\begin{array}{l}1550 \\
1300 \\
1700 \\
1300\end{array}$ & $\begin{array}{r}1 \\
20 \\
1 \\
20\end{array}$ & $\begin{array}{l}5 \cdot 1 \\
5 \cdot 8\end{array}$ & $\begin{array}{l}\text { B40s, Bw, xvw, AlOvw } \\
\text { B40vs, Bw, Sw, Aw, avvw } \\
\text { B30vs, Bvw, xvvw, AlOvvw } \\
\text { B30vs, Sw, Aw, Bvw }\end{array}$ & \\
\hline
\end{tabular}

Annealed at $1300^{\circ} \mathrm{C}$; all samples had a weight loss of $<1 \%$.

- Using pre-synthesized compounds to make the composition.

$\alpha, \alpha-\mathrm{Si}_{3} \mathrm{~N}_{4} ; \mathrm{B}, \beta-\mathrm{Si}_{3} \mathrm{~N}_{4} ; \mathrm{B3O}, \beta^{\prime}$-sialon $(Z=2 \cdot 18) ; \mathrm{B} 40, \beta^{\prime}$-sialon $(Z=2 \cdot 82) ; \mathrm{B} 60, \beta^{\prime}$-sialon $(Z=4 \cdot 0) ; \mathrm{CA}, \mathrm{CaO} . \mathrm{Al} \mathrm{O}_{2} \mathrm{O}_{3} ; \mathrm{C} 2 \mathrm{~A}$, $\mathrm{CaO} .2 \mathrm{Al}_{2} \mathrm{O}_{3} ; \mathrm{C} 6 \mathrm{~A}$ : CaO.6Al $\mathrm{O}_{3} ; \mathrm{AlO}, \mathrm{Al}_{2} \mathrm{O}_{3} ; \mathrm{SNO}, \mathrm{Sl}_{2} \mathrm{~N}_{2} \mathrm{O} ; \mathrm{X} 1, \mathrm{X}_{1}$ - phase; $\mathrm{S}, \mathrm{S}$-phase $\left(\mathrm{CaO} .1 \cdot 33 \mathrm{Al}_{2} \mathrm{O}_{3} \cdot 0 \cdot 67 \mathrm{Si}_{2} \mathrm{~N}_{2} \mathrm{O}\right) ; \mathrm{S}^{\prime}, \mathrm{Solid}$ solution between $\mathrm{S}$ and $\mathrm{CaO} .2 \mathrm{Al}_{2} \mathrm{O}_{3} ; \mathrm{C}, \alpha$-cristobalite $\left(\mathrm{SiO}_{2}\right) ; \mathrm{A}$, anorthite $\left(\mathrm{CaO} . \mathrm{Al}_{2} \mathrm{O}_{3} .2 \mathrm{SiO}_{2}\right) ; \mathrm{G}$, gehlenite $\left.\left(2 \mathrm{CaO} . \mathrm{Al}_{2} \mathrm{O}_{3} . \mathrm{SiO}\right)_{2}\right)$.

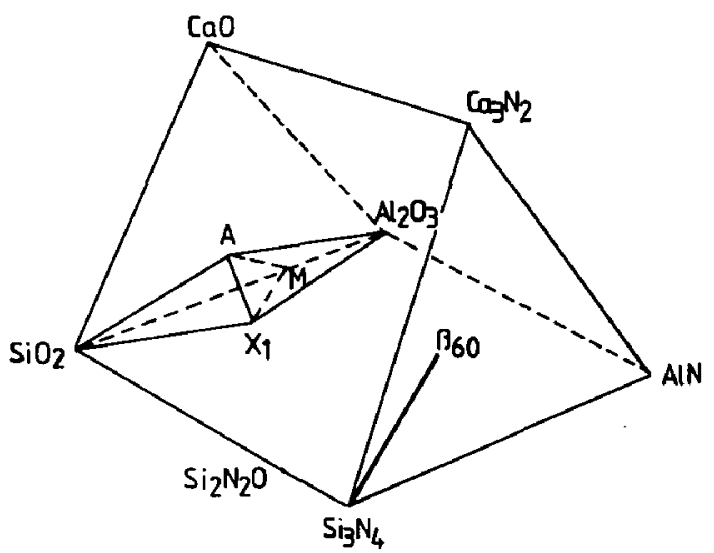

Fig. 2. Compatibility tetrahedra $\mathrm{SiO}_{2}$-anorthite- $\mathrm{X}_{1}-$ mullite and anorthite- $\mathrm{X}_{1}-$ mullite- $\mathrm{Al}_{2} \mathrm{O}_{3}$.

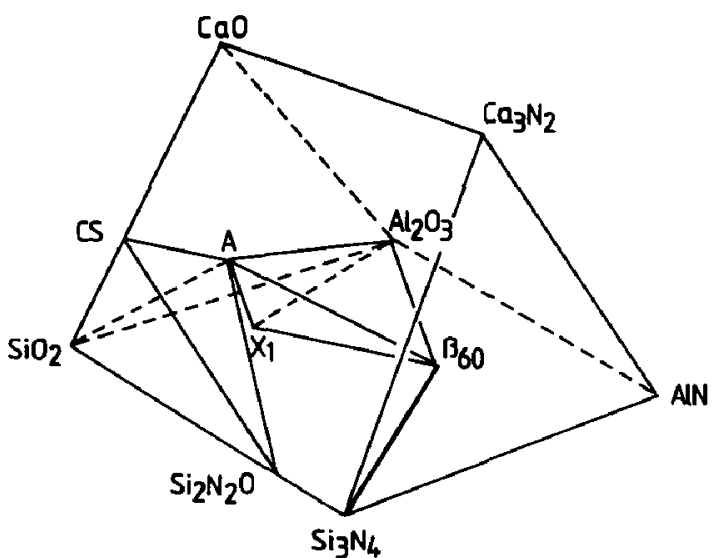

Fig. 3. Compatibility tetrahedra $\mathrm{SiO}_{2}-\mathrm{CaSiO}_{3}$-anorthite$\mathrm{Si}_{2} \mathrm{~N}_{2} \mathrm{O}$ and anorthite $-\mathrm{X}_{1}-\mathrm{B}_{60}-\mathrm{Al}_{2} \mathrm{O}_{3}$. 


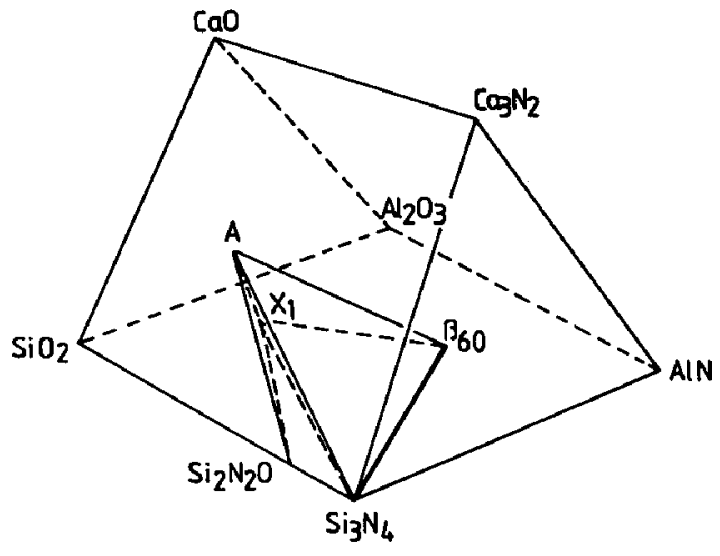

Fig. 4. Compatibility tetrahedra $\mathrm{Si}_{2} \mathrm{~N}_{2} \mathrm{O}$-anorthite- $\mathrm{X}_{1}-$ $\mathrm{Si}_{3} \mathrm{~N}_{4}$ and $\mathrm{Si}_{3} \mathrm{~N}_{4}-\mathrm{X}_{1}$-anorthite- $\beta_{60}$.

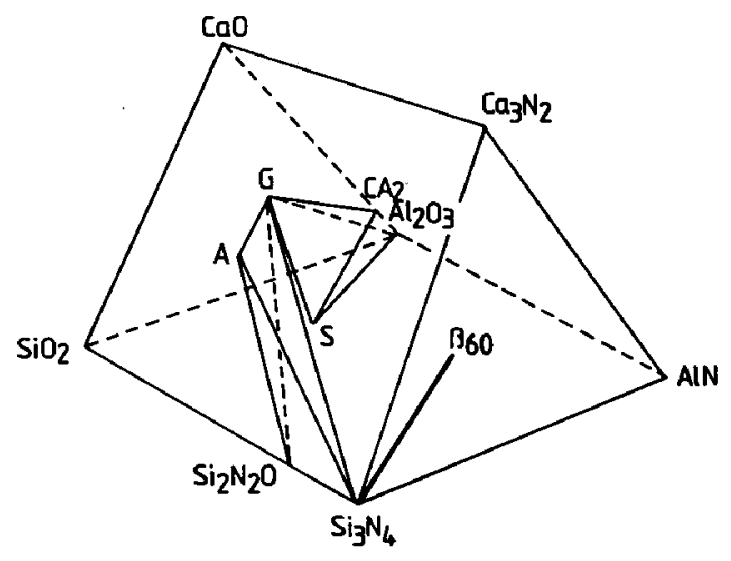

Fig. 6. Compatibility tetrahedra, $\mathrm{Si}_{2} \mathrm{~N}_{2} \mathrm{O}$-anorthitegehlenite- $\mathrm{Si}_{3} \mathrm{~N}_{4}$ and S-gehlenite-CaO $2 \mathrm{Al}_{2} \mathrm{O}_{3}-\mathrm{Al}_{2} \mathrm{O}_{3}$.

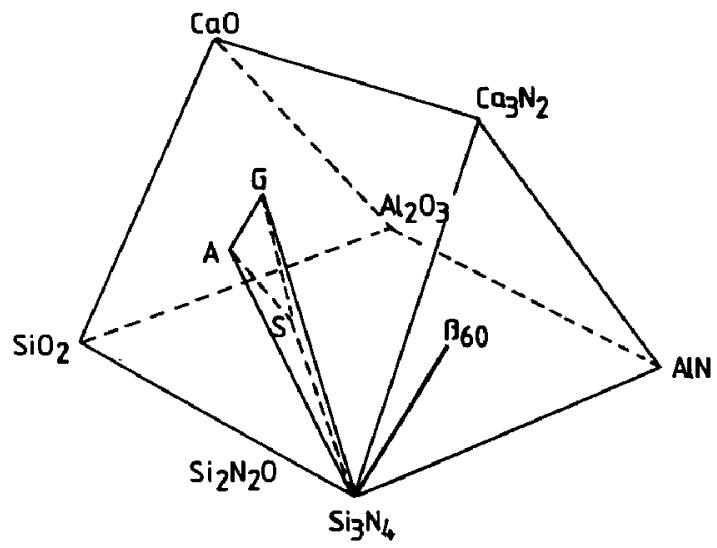

Fig. 8. Compatibility tetrahedron $\mathrm{Si}_{3} \mathrm{~N}_{4}$-S-anorthitegehlenite.

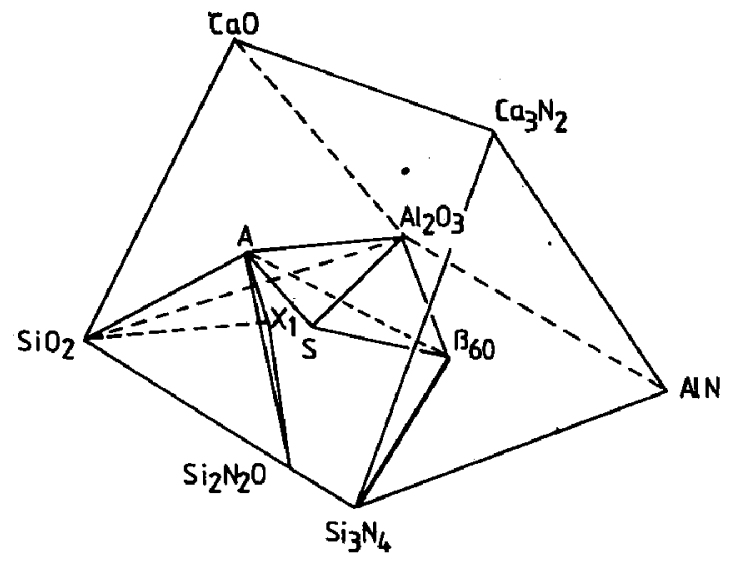

Fig. 5. Compatibility tetrahedra $\mathrm{Si}_{2} \mathrm{~N}_{2} \mathrm{O}-\mathrm{SiO}_{2}-\mathrm{X}_{1}$-anorthite and $\mathrm{S}$-anorthite- $\mathrm{Al}_{2} \mathrm{O}_{3}-\beta_{60}$.

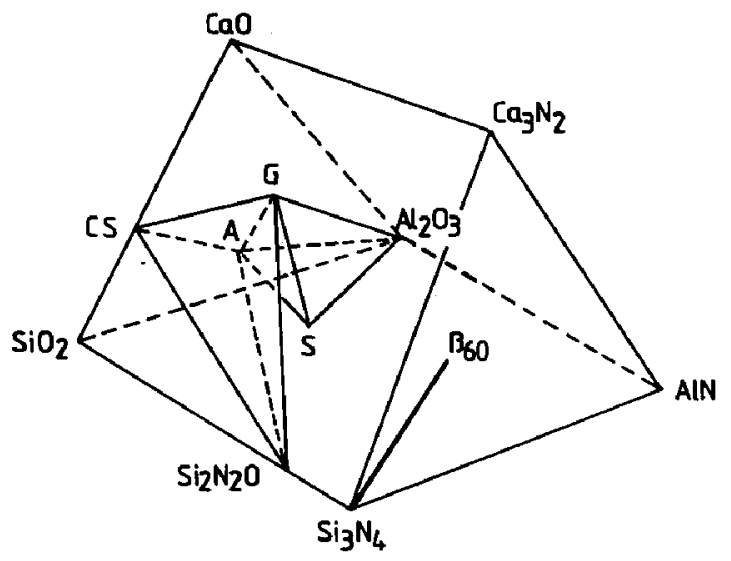

Fig. 7. Compatibility tetrahedra $\mathrm{Si}_{2} \mathrm{~N}_{2} \mathrm{O}-\mathrm{CaSiO}_{3}$-anorthitegehlenite and $\mathrm{S}$-anorthite-gehlenite- $\mathrm{Al}_{2} \mathrm{O}_{3}$.

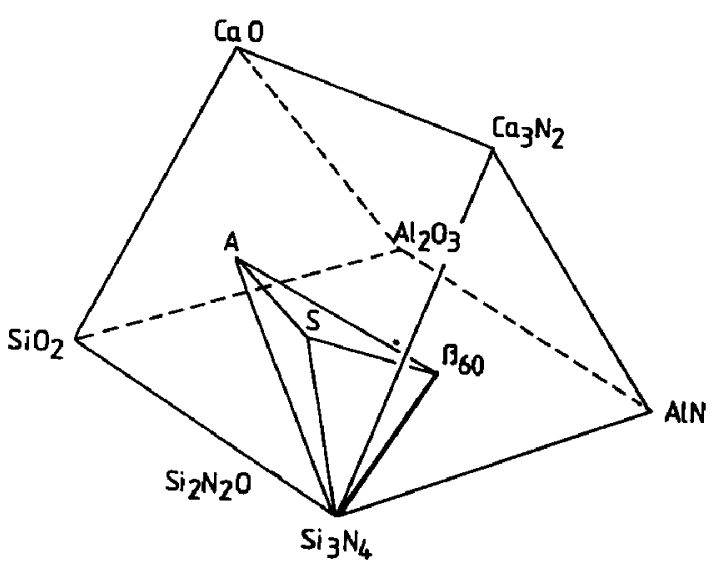

Fig. 9. Compatibility tetrahedron $\mathrm{Si}_{3} \mathrm{~N}_{4}-\beta_{60}-\mathrm{S}$-anorthite. 
Table 2. Quaternary compatibility relationships in the system $\mathrm{Si}, \mathrm{Al}, \mathrm{Ca} / \mathrm{N}, \mathrm{O}$

$\mathrm{X}_{1}-\mathrm{SiO}_{2}$-anorthite-mullite

(Fig. 2)

$\mathrm{X}_{1}$-anorthite-mullite- $\mathrm{Al}_{2} \mathrm{O}_{3}$

(Fig. 2)

$X_{1}$-anorthite $-\mathrm{Al}_{2} \mathrm{O}_{3}-\beta_{60}$

$X_{1}$-anorthite- $\beta_{\mathrm{BO}}-\mathrm{Si}_{3} \mathrm{~N}_{4}$

$X_{1}$-anorthite- $\mathrm{Si}_{3} \mathrm{~N}_{2}-\mathrm{Si}_{2} \mathrm{~N}_{2} \mathrm{O}$

$X_{1}$-anorthite- $\mathrm{Si}_{2} \mathrm{~N}_{2} \mathrm{O}-\mathrm{SiO}_{2}$

Anorthite- $\mathrm{Si}_{2} \mathrm{~N}_{2} \mathrm{O}-\mathrm{SiO}_{2}-\mathrm{CaSiO}_{3}$

Anorthite- $\mathrm{Si}_{2} \mathrm{~N}_{2} \mathrm{O}-\mathrm{CaSiO}_{3}$-gehlenite

Anorthite- $\mathrm{Si}_{2} \mathrm{~N}_{2} \mathrm{O}$-gehlenite- $\mathrm{Si}_{3} \mathrm{~N}_{4}$

$\mathrm{S}$-anorthite- $\mathrm{Al}_{2} \mathrm{O}_{3}-\mathrm{B}_{60}$

S- $\mathrm{Al}_{2} \mathrm{O}_{3}-\mathrm{CaO} .2 \mathrm{Al}_{2} \mathrm{O}_{3}$-gehlenite

$\mathrm{S}-\mathrm{Al}_{2} \mathrm{O}_{3}$-gehlenite-anorthite

S-gehlenite-anorthite- $\mathrm{Si}_{3} \mathrm{~N}_{4}$

S-anorthite- $\mathrm{Si}_{3} \mathrm{~N}_{6}-\beta_{60}$

(Fig. 3)

(Fig. 4)

(Fig. 4)

(Fig. 5)

(Fig. 3)

(Fig. 7)

(Fig. 6)

(Fig. 5)

(Fig. 6)

(Fig. 7)

(Fig. 8)

(Fig. 9)

system even using synthesized $\mathrm{CaO} \cdot 6 \mathrm{Al}_{2} \mathrm{O}_{3}$ (including a small amount of $\mathrm{Al}_{2} \mathrm{O}_{3}$ ) as a starting material to form the compositions. The composition regions where $\mathrm{CaO} .6 \mathrm{Al}_{2} \mathrm{O}_{3}$ should appear always showed $\mathrm{Al}_{2} \mathrm{O}_{3}$ and/or $\mathrm{CaO} .2 \mathrm{Al}_{2} \mathrm{O}_{3}$ instead. $\mathrm{CaO} .6 \mathrm{Al}_{2} \mathrm{O}_{3}$ is an incongruent melting compound ${ }^{4}$ and was reported as unstable in some early work. ${ }^{5}$ Because of its absence in the system $\mathrm{Si}, \mathrm{Al}, \mathrm{Ca} / \mathrm{N}, \mathrm{O}$ as studied, $\mathrm{CaO} .6 \mathrm{Al}_{2} \mathrm{O}_{3}$ was not taken into account in establishing the compatibility phase relationships.

The compositions located in the region bounded by $\mathrm{Si}_{3} \mathrm{~N}_{4}, \mathrm{~S}$-phase, $2 \mathrm{CaO} \cdot \mathrm{Al}_{2} \mathrm{O}_{3} \cdot \mathrm{SiO}_{2}, \mathrm{CaO}$. $\mathrm{Al}_{2} \mathrm{O}_{3}, \mathrm{Al}_{2} \mathrm{O}_{3}$ and $\beta_{60}$, where $\mathrm{AlN}$ or AlN polytypes appeared, were not used to establish compatibility tetrahedra, because they are beyond the region studied in this paper.

In some nitrogen-rich compositions with some proper contents of $\mathrm{Al}$ and $\mathrm{Ca}$ (compositions 9 and $10)$, the appearance of $\alpha^{\prime}$-sialon ${ }^{6}$ was expected and was also not taken into the establishment of phase relationships concerned.

The existence of the tetrahedron S-phaseanorthite- $\mathrm{Si}_{3} \mathrm{~N}_{4}-\beta_{60}$ indicates that, in a $\mathrm{Ca}-$ sialon system, no crystalline compound is suitable to be used as a refractory second grain boundary phase to generate a promising $\beta^{\prime}$-sialon ceramics, because of their relatively low melting points $\left(1550^{\circ} \mathrm{C}\right.$ for anorthite and $1450^{\circ} \mathrm{C}$ for S-phase).

\section{CONCLUSIONS}

Subsolidus phase relationships in the region bounded by $\mathrm{Si}_{3} \mathrm{~N}_{4}, \mathrm{SiO}_{2}, \mathrm{CaSiO}_{3}, 2 \mathrm{CaO} . \mathrm{Al}_{2} \mathrm{O}_{3}$. $\mathrm{SiO}_{2}, \mathrm{CaO} . \mathrm{Al}_{2} \mathrm{O}_{3}, \mathrm{Al}_{2} \mathrm{O}_{3}$ and $\beta_{60}$ have been established. In this region, a new phase (S-phase) with composition near to $\mathrm{CaO} \cdot 1 \cdot 33 \mathrm{Al}_{2} \mathrm{O}_{3}$. $0.67 \mathrm{Si}_{2} \mathrm{~N}_{2} \mathrm{O}$ and a continuous solid solution of the new phase with $\mathrm{CaO} .2 \mathrm{Al}_{2} \mathrm{O}_{3}$ was found. The existence of the compatibility tetrahedron $\mathrm{S}$ -

Table 3, X-ray pattern of the unknown $\mathbf{S}$-phase and its solid solution $\mathbf{S}_{\mathrm{ss}}$

\begin{tabular}{|c|c|c|c|c|c|}
\hline \multicolumn{2}{|c|}{$S$} & \multicolumn{2}{|c|}{$\mathrm{S}_{\mathrm{ss}}$ (sample 23) } & \multicolumn{2}{|c|}{$\mathrm{CaO} \cdot 2 \mathrm{Al}_{2} \mathrm{O}_{3}$ (ASTM) } \\
\hline$d^{s}$ & $\mid / l_{1}^{b}$ & d & $I / I_{1}$ & d & $1 / I_{1}$ \\
\hline $6 \cdot 18$ & vuw & $6 \cdot 25$ & vww & $\begin{array}{l}6 \cdot 16 \\
4 \cdot 616\end{array}$ & $\begin{array}{l}6 \\
6\end{array}$ \\
\hline $4 \cdot 34$ & $m$ & $4 \cdot 40$ & $w$ & 4.40 & 55 \\
\hline $3 \cdot 55$ & $w$ & 3.59 & vw & 3.60 & 20 \\
\hline $3 \cdot 47$ & vs & 3.50 & vs & $\begin{array}{l}3.50 \\
3.23\end{array}$ & $\begin{array}{r}100 \\
6\end{array}$ \\
\hline 3.09 & $\mathrm{~m}$ & $3 \cdot 10$ & $\mathbf{m}$ & 3.08 & 30 \\
\hline $2 \cdot 81$ & vw & $2 \cdot 79$ & vrw & $2 \cdot 882$ & 20 \\
\hline $2 \cdot 74)$ & & $2 \cdot 75$ & $s$ & $2 \cdot 753$ & 25 \\
\hline $2 \cdot 73\}$ & s & $2 \cdot 725$ & $\mathbf{s}$ & $2 \cdot 712$ & 25 \\
\hline $2 \cdot 61$ & $\mathbf{s}$ & $2 \cdot 61$ & $\mathbf{s}$ & 2.599 & 60 \\
\hline $2 \cdot 55$ & $\mathbf{s}$ & $2 \cdot 58$ & $\mathbf{s}$ & & \\
\hline 2.52 & $\mathrm{mw}$ & $2 \cdot 53$ & $w$ & $\begin{array}{l}2 \cdot 534 \\
2 \cdot 462\end{array}$ & $\begin{array}{r}15 \\
6\end{array}$ \\
\hline $2 \cdot 42$ & $w$ & $2 \cdot 42$ & $w$ & $\begin{array}{l}2 \cdot 452 \\
2 \cdot 436\end{array}$ & $\begin{array}{r}6 \\
16\end{array}$ \\
\hline $2 \cdot 34$ & $w$ & $2 \cdot 33$ & $w$ & $\begin{array}{l}2 \cdot 404^{\circ} \\
2 \cdot 350\end{array}$ & $\begin{array}{r}6 \\
16\end{array}$ \\
\hline $2 \cdot 20$ & vw & $2 \cdot 19$ & vw" & $2 \cdot 181$ & 6 \\
\hline 2.06 & $w$ & $2 \cdot 06$ & $w$ & $2 \cdot 054$ & 16 \\
\hline
\end{tabular}

a $d$ is the spacing in the lattice parameter.

- $\mathrm{I} / \mathrm{l}$, is the relative intensity of $\mathrm{X}$-ray diffraction lines. 
Table 4. Compositions and firing conditions explored for the preparation of pure S-phase

\begin{tabular}{|c|c|c|c|c|c|c|c|c|}
\hline \multirow[t]{2}{*}{ No. } & \multicolumn{5}{|c|}{ Composition (at\%) } & \multirow{2}{*}{$\begin{array}{l}\text { Firing } \\
\text { condition } \\
\left({ }^{\circ} \mathrm{C}-\mathrm{h}\right)\end{array}$} & \multirow{2}{*}{$\begin{array}{l}\text { Weight } \\
\text { loss. } \\
(\%)\end{array}$} & \multirow[t]{2}{*}{ Phase present } \\
\hline & $\mathrm{Ca}$ & Al & Si & $\mathbf{N}$ & 0 & & & \\
\hline 1 & 1 & 2 & 2 & 2 & 5 & $\begin{array}{c}1320-0.5 \\
1380-0.5 \\
1450-1 \\
\text { d. } 1200-45\end{array}$ & $\begin{array}{l}1 \cdot 8 \\
1 \cdot 8 \\
2 \cdot 0 \\
-\end{array}$ & $\begin{array}{l}\text { Svs; Gs; } \alpha m \\
\alpha m ; 12 H w \\
\alpha w ; 12 H w \\
S m ; G m^{*}\end{array}$ \\
\hline 2 & 1 & 2 & 2 & $2 \cdot 33$ & $4 \cdot 5$ & $\begin{array}{l}1550-1+1650-1 \\
\text { d. } 1200-45\end{array}$ & $\frac{3.7}{-}$ & $\begin{array}{l}\alpha v w ; \text { Pvw. } \\
\text { Ss; Gs; } \alpha v w ; \text { Pvw }\end{array}$ \\
\hline 3 & 1 & 2 & 2 & $2 \cdot 66$ & 4 & 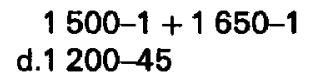 & $\begin{array}{l}0.9 \\
-\end{array}$ & $\begin{array}{l}\alpha^{\prime} w ; \text { Pvw } \\
\text { Sm;Sm; } \alpha w ; P v w\end{array}$ \\
\hline 4 & 1 & $2 \cdot 66$ & $1 \cdot 34$ & $1 \cdot 34$ & $5 \cdot 66$ & $\begin{aligned} 1400-0.5 \\
\text { d.1 } 400-48 \\
1450-1 \\
1550-0.5 \\
\text { d.1 } 200-45\end{aligned}$ & $\begin{array}{l}\frac{3 \cdot 3}{3 \cdot 2} \\
- \\
-\end{array}$ & $\begin{array}{l}\text { Svs; } \alpha^{\prime} \text { tr. } \\
\text { Svs }{ }^{b} \\
15 R w ; 12 H v w ; \alpha t r . \\
\text { none } \\
\text { Ss; Gs }\end{array}$ \\
\hline 5 & 1 & $2 \cdot 4$ & $1 \cdot 6$ & $1 \cdot 6$ & $5 \cdot 4$ & $\begin{array}{l}1300-0.5 \\
1380-0.5\end{array}$ & $\begin{array}{l}1.9 \\
1.7\end{array}$ & $\begin{array}{l}\text { Svs; Gs; } \alpha m \\
12 \mathrm{Hw}\end{array}$ \\
\hline
\end{tabular}

$\mathrm{S}, \mathrm{S}$-phase; G, gehlenite; $\alpha, \alpha-\mathrm{Si}_{3} \mathrm{~N}_{4} ; \alpha^{\prime}, \alpha^{\prime}$-sialon; P, AIN-polytype.

- Phase assemblages were the same as devitrified at $1100^{\circ} \mathrm{C}$ and $1300^{\circ} \mathrm{C}$.

${ }^{b} \mathrm{X}$-ray diffraction lines became sharper; $1200^{\circ} \mathrm{C}$ and $1300^{\circ} \mathrm{C}$ had no such effect.

phase-anorthrite- $\beta-\mathrm{Si}_{3} \mathrm{~N}_{4}-\beta_{60}$ implies that the fabrication of $\beta^{\prime}$-sialon ceramics with a $\mathrm{Ca}$ containing compound as a possible second crystalline grain boundary phase cannot be promising for high temperature applications.

\section{REFERENCES}

1. NAIK, I. K. \& TIEN, T. Y., J. Am. Ceram. Soc., 62(11-12) (1979) 642.
2. SUN, W. Y., HUANG, Z. K. \& CHENG, J. X., Trans. Brit. Ceram. Soc., 82 (1983) 173.

3. SUN, W. Y., HUANG, Z. K., CAO, G. Z. \& YAN, D. S., Scientia Sinica (Series A), XXXI(6) (1988) 742.

4. LEVIN, E. M., ROBBINS, C. K. \& MCMURDIE, H. F. (Eds), Phase Diagram for Ceramists, The American Ceramic Society, 1964.

5. GENTILE, A. L. \& FOSTER, W. R., J. Am. Ceram. Soc., 46(2) (1963) 74.

6. HAMPSHIRE, S., PARK, H. K., THOMPSON, D. P. \& JACK, K. H., Nature, 274(5674) (1978) 880. 